\begin{tabular}{|c|}
\hline Pbilosophia \\
Scientic \\
\hline
\end{tabular}

\section{Philosophia Scientiæ}

Travaux d'histoire et de philosophie des sciences

$11-2$ | 2007

Varia

\title{
Méthode axiomatique et négation chez Hilbert
}

\section{Eric Audureau}

\section{OpenEdition}

\section{Journals}

Édition électronique

URL : http://journals.openedition.org/philosophiascientiae/335

DOI : 10.4000/philosophiascientiae.335

ISSN : 1775-4283

Éditeur

Éditions Kimé

Édition imprimée

Date de publication : 1 novembre 2007

Pagination : $67-96$

ISBN : 978-2-84174-439-8

ISSN : $1281-2463$

\section{Référence électronique}

Eric Audureau, « Méthode axiomatique et négation chez Hilbert », Philosophia Scientiæ [En ligne], 11-2 | 2007, mis en ligne le 30 juin 2011, consulté le 15 janvier 2021. URL : http://journals.openedition.org/ philosophiascientiae/335; DOI : https://doi.org/10.4000/philosophiascientiae.335 


\title{
Méthode axiomatique et négation chez Hilbert
}

\author{
Eric Audureau \\ Université de Provence \\ CEPERC- UMR 6059 CNRS
}

\section{Résumé :}

a) La doctrine de la connaissance défendue par Hilbert au cours du développement de la théorie de la démonstration est constituée dès la Conférence de Paris de 1900. Elle précède donc la théorie de la démonstration.

b) L'application du principe fondamental de l'épistémologie hilbertienne ( «Au commencement est le signe ») à la caractérisation de la négation logique est l'un des problèmes principaux de la théorie de la démonstration.

c) Pour pouvoir caractériser la négation en termes de manipulation de signes, il faut abandonner la méthode axiomatique et donc sacrifier l'essentiel de la doctrine hilbertienne de la connaissance.

\section{Abstract:}

a) The epistemology advocated by Hilbert through the development of proof theory is already held in his Paris Address in 1900.

b) The application of the fondamental principle of Hilbert's epistemology (am Anfang ist das Zeichen) to the characterization of logical negation is one of the main problems of Hilbert's proof theory

c) In order to characterize negation a property of signs one has to give up the axiomatic method, namely, the core of Hilbert's epistemology.

\section{Introduction}

Je me propose d'analyser ici la conception des mots logiques développée par Hilbert dans le cadre de la théorie de la démonstration, ceci 
en l'examinant sous le double aspect de la méthode axiomatique et de l'épistémologie hilbertienne et en me concentrant sur la négation ${ }^{1}$.

Lorsqu'on étudie une question aussi spécifique, on est inévitablement conduit à l'isoler d'un ensemble de problèmes auxquels elle est nécessairement liée. Par exemple, en toute rigueur, la négation est liée à la quantification. Et, chez Hilbert, la construction de la quantification, par le biais de l'opérateur de choix, est indissociable de celle de l'analyse et, donc, de l'ensemble des questions techniques, épistémologiques et ontologiques soulevées par la théorie hilbertienne de la démonstration. Comme il ne peut être question, dans le cadre d'un simple article, de traiter de l'ensemble de ces questions et de leurs rapports, je formulerai, à titre introductif, quelques remarques préliminaires fixant les bornes à l'intérieur desquelles la nature de la négation est traitée et résumant ce qui est tenu pour acquis dans le cadre de cette brève étude. Pour la raison juste dite, je n'entrerai évidemment pas dans la justification du contenu de ces remarques préliminaires.

Je présenterai le plan de cette étude à la fin de ces remarques introductives.

\section{Remarques préliminaires à l'étude du pro- blème de la négation chez Hilbert.}

1.1 On sait que les recherches de Hilbert sur les fondements des mathématiques se distinguent de celles de ses contemporains par le fait que celui-ci se propose de fonder simultanément la logique et l'arithmétique. Ce qui revient à construire indépendamment les mots logiques et le concept de nombre. Hilbert s'opposait ainsi, d'une part, à ceux qui, comme Frege, Russell ou Hanh, voulaient déduire le concept de nombre à partir des concepts de la logique et, d'autre part, à ceux qui, comme Poincaré ou Brouwer, jugeant la logique stérile, estimaient que seule l'origine du concept de nombre était redevable d'une justification épistémologique.

Le second aspect de ce programme de Hilbert, celui concernant l'arithmétique, est souvent commenté, principalement à cause de l'ambitieux objectif de démontrer la cohérence des mathématiques. Par contre, le premier aspect, le projet de construire les mots logiques, est rarement

\footnotetext{
${ }^{1}$ Je suis redevable à un examinateur anonyme de la première version de cet article dont certaines des observations m'ont invité à essayer d'en améliorer la forme.
} 
étudié; et lorsqu'il l'est, les commentateurs se concentrent uniquement sur l'analyse des quantificateurs.

1.2. Il est clair que la seule considération du traitement de la quantification nous place sur un terrain d'analyse qui manque de fermeté. En effet, chez Hilbert, les concepts de quantification et de fonction sont 《 finitisés » à l'aide du même procédé, c'est-à-dire par l'emploi de la fonction $\varepsilon$ qui, pour fixer les idées, repose sur un principe général dont le contenu est analogue à celui de l'axiome du choix. De sorte que ce qui est spécifique à l'approche de Hilbert, derechef distinguer la construction de l'arithmétique et celle de la logique, disparaît ou, plus exactement, semble se confondre. On le constate en comparant cette approche à celle qui, du point de vue épistémologique, s'y apparente le plus : l'empirisme logique.

A s'en tenir à la seule considération des quantificateurs, il n'y aurait plus de raison, à vrai dire, de distinguer la position de Hilbert (sur la logique) de celle de l'empirisme logique. Le représentant le plus autorisé de l'empirisme logique ${ }^{2}$, Hans Hanh, reconnaissait que l'axiome du choix s'oppose au caractère tautologique des autres principes logiques [Hanh 1980, 112], tels que le principe de contradiction [Hanh 1980, 42], car il « paraît avoir un contenu réel » [Hanh 1980, 34] le rendant irréductible à la forme purement linguistique requise pour toutes les propositions de la logique. Devoir justifier à deux titres, pour la logique et pour l'arithmétique, l'usage du même principe de choix devrait donc faire écho à une confusion dans les principes épistémologiques sur lesquels repose la connaissance mathématique. Et Hanh relève bien deux défauts dans la démarche de Hilbert. $1^{\circ}$ ) En subordonnant le concept de nombre cardinal à celui de nombre ordinal, elle viole l'ordre des raisons en construisant le simple à partir du composé ${ }^{3} .2^{\circ}$ ) En posant le primat de l'arithmétique sur la géométrie, Hilbert fait non seulement un choix dogmatique et arbitraire, mais il souscrit également, à son corps défendant, à un vestige d'idéalisme en prêtant à l'intuition arithmétique le statut de l'évidence [Hanh 1980, 67].

Il faudrait par conséquent constamment fermer les yeux sur des questions omniprésentes d'épistémologie et de théorie des ensembles pour

\footnotetext{
${ }^{2}$ Hans Hahn fut, avec O.Neurath et P.Frank, non seulement l'un des co-fondateurs, mais doit, encore plus, selon le témoignage de Frank, «être considéré comme le véritable fondateur du Cercle de Vienne » (Cité par K. Menger [Hanh 1980, X]).

${ }^{3}$ Le concept de cardinal est une caractéristique invariante d'un ensemble pour toutes les projections injectives, tandis que celui d'ordinal n'est invariant que pour les projections isomorphes conservant la structure d'ordre des ensembles, [Hanh 1980, $60]$.
} 
continuer à voir la trace du dualisme logique-arithmétique dans les recherches hilbertiennes sur la quantification.

1.3 Dans ces conditions, pour examiner le programme de construction des mots logiques, il est préférable de suivre les indications données explicitement par Hilbert dès la première ébauche, en 1904, de la théorie de la démonstration où il s'essaye à définir la négation, le mot logique non. D'autant que, comme nous le verrons, cette intention initiale est présente d'une façon tout aussi explicite en 1922 quand Hilbert entreprend réellement de développer la théorie de la démonstration.

L'intérêt de l'étude de cette conception de la négation est de permettre de constater que les recherches de Hilbert en la matière préfigurent une théorie générale philosophiquement neutre des mots logiques. Celle-ci sera élaborée par son élève Gentzen avec le calcul des séquents et généralisée à partir d'une étude de Dana Scott beaucoup plus tardive, [Scott 71].

Hilbert avait à sa disposition tous les moyens pour développer luimême la théorie élaborée par Gentzen. Deux obstacles l'ont empêché d'y parvenir. Le premier est son attachement à la méthode axiomatique en général. Le deuxième est sa défiance à l'égard de l'intuitionnisme dans laquelle un ressentiment personnel à l'égard de Brouwer vient se mêler à des convictions philosophiques très discutables. Ce préjugé se traduit directement dans la conception de la méthode axiomatique propre à Hilbert.

1.4. La distinction entre méthode axiomatique en général et conception de la méthode axiomatique propre à Hilbert est indispensable pour apprécier correctement l'apport positif de Hilbert à la théorie générale des mots logiques de Gentzen et Scott.

On pense parfois que la méthode axiomatique n'est qu'une méthode et qu'elle n'engage pas philosophiquement ${ }^{4}$. Il est exact que, dans la perspective des fondements des mathématiques, Hilbert a défendu cette position, mais on sait que ce point de vue n'a jamais fait l'unanimité. La correspondance entre Frege et Hilbert, [Rivenc \& Rouilhan 1992, 220235] ou la critique virulente de Padoa au sujet du second problème de Hilbert, [Padoa 1902], l'attestent. Pour ces adversaires de la conception hilbertienne de l'axiomatique, il ne peut y avoir de démonstration directe de la cohérence d'un système d'axiomes, celle-ci ne pouvant être obtenue que par l'exhibition d'un modèle. Les axiomes ne définissent pas leur référence, mais ils postulent l'existence de celle-ci et en dépendent.

\footnotetext{
${ }^{4}$ C'est, par exemple, l'opinion d'un des rapporteurs sur la première version du présent article, et ce cas n'est pas isolé.
} 
Alors que pour Hilbert la méthode axiomatique permet de définir, ou d'introduire, les objets dont elle étudie les propriétés, Frege estime que les objets existent préalablement à la description qu'en donne les théories axiomatiques et que celles-ci doivent se conformer à cette existence.

Hilbert n'a pas donné suite à la proposition de Frege de publier leur correspondance. Il n'a pas non plus répondu à Padoa malgré la sollicitation pressante de celui-ci. Faut-il en conclure que ces derniers livraient un combat d'arrière-garde et que Hilbert ne s'est pas soucié de poursuivre la controverse à cause du caractère archaïsant de la position de ses adversaires?

On pourrait le croire lorsqu'on lit Bourbaki : «... les mots "il existe" dans un texte formalisé n'ont pas plus de "signification" que les autres et il n'y a pas à considérer d'autres types "d'existence" dans les démonstrations formalisées », [Bourbaki 1960, 57]. Mais les progrès de la logique mathématique ont montré que Frege et Padoa avaient raison. Le théorème de complétude de la logique classique du premier ordre suffit pour le constater. Ce théorème exige, à titre préliminaire, qu'aux côtés de notions définies à partir de combinaisons spatiales de symboles, on introduise des notions qui dépendent de la signification [Gödel 29, 66]. Cette leçon est confirmée a fortiori par le théorème d'existence de propositions d'arithmétique formellement indécidables. Certaines de ces propositions pouvant exprimer leur propre indécidabilité [Gödel 31], elles sont donc vraies selon un concept de vérité qui ne peut être saisi par la formalisation de l'axiomatique.

Frege, Padoa ou Gödel n'ont jamais remis en cause la méthode axiomatique en général, mais ils ont critiqué, et cette critique est irrémédiable avec les deux théorèmes Gödel juste cités, la conception hilbertienne de l'axiomatique dont toute la substance est uniquement symbolique ${ }^{5}$.

La méthode axiomatique est donc dogmatique : elle pose de facto une disjonction entre les états de choses, ce dont est fait l'interprétation ou le modèle, et les descriptions de ces états de choses données par les axiomes $^{6}$. Elle engage donc philosophiquement, même si, par elle-même,

\footnotetext{
${ }^{5}$ Lorsque Gödel [Gödel 31, 194] souligne que le second théorème d'incomplétude ne contredit pas le point de vue de Hilbert selon lequel il est possible de donner une démonstration finitiste de cohérence (car « il est concevable qu'il puisse exister des démonstrations finitistes qui ne peuvent pas être exprimées dans le formalisme [de l'arithmétique ou de la théorie des ensembles] »), il veut simplement dire que le programme de Hilbert peut être poursuivi à condition que l'on renonce à l'idée que la méthode axiomatique soit exclusivement une méthode formelle qui dispense de poser l'existence des choses (faites d'un autre matériau que de symboles).

${ }^{6}$ Sauf dans les cas triviaux où l'axiomatisation est superflue, c'est-à-dire lorsqu'il existe un procédé de décision pour la validité des expressions du système comme c'est,
} 
elle ne détermine pas univoquement un système philosophique. L'erreur de Hilbert est d'avoir cru que la méthode axiomatique pouvait être une philosophie critique ${ }^{7}$.

1.5. Le ressort principal de la méthode axiomatique hilbertienne, dans le cadre des fondements des mathématiques, est de placer la connaissance des signes, et de leur manipulation, au principe de la connaissance des choses (des objets de la logique, de l'arithmétique et de l'analyse). Sans se demander si, en son for intérieur, Hilbert était réellement formaliste $^{8}$ ou, ce qui serait encore plus hors de propos, invoquer, qu'en géométrie, il donne des preuves de cohérence par la méthode des interprétations (comme s'il en existait d'autre!), il faut constater, après Cavaillès, que puisque le signe est l'élément commun à la logique et aux mathématiques alors, chez Hilbert, « l'axiomatisation s'achève nécessairement en formalisation » [Cavaillès 1938, 90].

1.6. Le formalisme ou, mieux, le sémiologisme de Hilbert était la bonne voie pour parvenir à une théorie générale des mots logiques qui soit indépendante de toute doctrine philosophique et des doctrines dogmatiques en particulier. Cependant, l'attachement de Hilbert à la méthode axiomatique s'opposait à ce développement. Mais, ici, c'est la méthode axiomatique en général qui est en cause et non pas la forme particulière, anti-dogmatique et purement symbolique, que souhaitait lui donner Hilbert. En d'autres termes, tous les partisans de la méthode axiomatique en logique, à cause du caractère dogmatique de cette méthode, sont ici concernés. En effet, la méthode des séquents de Gentzen, qui permet de construire la théorie générale des mots logiques, est faite uniquement de règles d'inférence. C'est cet aspect qui lui permet d'exprimer dans un cadre unitaire, à partir d'un nombre restreint et fixé de règles, tous les systèmes logiques concevables, pourvu que ceux-ci satisfassent à des conditions d'identité très générales ${ }^{9}$.

par exemple, le cas pour le calcul des propositions.

${ }^{7} \mathrm{Je}$ précise que le mot dogmatique (de même que critique) est employé au sens philosophique du terme puisqu'un rapporteur sur la première version de cet article l'a compris en son sens usuel et péjoratif. J'en fais ici le même usage que [Vuillemin 1986]. Il y a plusieurs types de systèmes philosophiques dogmatiques et plusieurs types de système critiques (ou de l'examen, comme les appelle Vuillemin). Les théorèmes de Gödel cités ont également une incidence sur certains systèmes dogmatiques. Par exemple, sur celui de l'empirisme logique évoqué dans la remarque 1.2 ci-dessus.

${ }^{8}$ Hilbert n'est pas formaliste au moins en deux sens. 1) Lorsqu'on compare l'éclectisme de sa doctrine au formalisme authentique de [Curry 1977], Cf. infra, section 3. 2) Lorsqu'on compare son attitude à celle de von Neumann. Ce dernier, en formaliste conséquent avec lui-même, estimait, qu'après les théorèmes de Gödel, il fallait renoncer à fonder les mathématiques [Mancosu 1999, 40].

${ }^{9}$ Les logiques dites non-monotones ne répondent pas à ces conditions d'identité. 
Nous verrons que Gentzen, pour une large part, n'a eu qu'à recoder dans le cadre du calcul des séquents des conditions générales sur la relation de déduction que Hilbert avait d'abord exprimé sous forme d'axiomes et qui, de ce fait, ne valait que pour la logique classique.

Je restituerai d'abord la doctrine de la connaissance de Hilbert. J'analyserai ensuite brièvement ses traits paradoxaux (section 3). Dans la section 4, consacrée à l'examen du traitement hilbertien de la négation, je montrerai que, dès la mise en œuvre du programme de Hilbert, les aspects contradictoires de cette doctrine se traduisent immédiatement par une suite de reculades. Dans la section 5 je montre pourquoi il faut renoncer à la méthode axiomatique pour que la part positive du programme de Hilbert puisse apparaître. Enfin, je signale, dans une brève conclusion, les conséquences fâcheuses de deux cas d'hilbertisme tardif dans la pensée contemporaine, l'un emprunté à la vie philosophique, l'autre à la vie scientifique.

\section{Epistémologie et universalité de la méthode axiomatique}

On n'a pas assez remarqué que Hilbert avait adopté la plupart des points de sa doctrine dès la conférence de Paris de 1900 [Hilbert 1902]. Peut-être est-ce parce que les considérations exposées dans celle-ci paraissent inspirées par une analyse immanente de la pratique mathématique, dans l'esprit de Hadamard ou du Poincaré de L'intuition et la logique en mathématiques [Poincaré 1905, 27-40] plutôt que par des considérations relevant des fondements des mathématiques, ceci même si les Premier et Second problèmes ${ }^{10}$ les orientent inévitablement dans cette dernière direction.

Notons d'abord, contre certaines interprétations récentes, que Hilbert n'est pas instrumentaliste. La Physique, il est vrai, fournit un grand nombre de questions aux mathématiciens, mais à l'origine de la plupart des problèmes de l'analyse et de la théorie des nombres nous trouvons des questions que l'esprit humain s'adresse à lui même, [Hilbert 1902, 5]. Ce que poursuivent les mathématiciens avec l'examen de ces problèmes, c'est

\footnotetext{
Mais dans ces «logiques », on confond sans cesse ce qui relève de la logique, c'est-à-dire de l'étude du raisonnement correct, et ce qui relève d'un ordre de connaissance tout à fait différent, comme l'analyse d'énoncés hypothétiques dont le contenu empirique peut être provisoire.

${ }^{10}$ Respectivement, démontrer l'hypothèse du continu et démontrer la cohérence de l'arithmétique.
} 
« un besoin philosophique général de notre entendement (Verstand) » à savoir la recherche de la rigueur dans la démonstration [ibid., 6]. La rigueur doit être identifiée au finitisme des déductions logiques [ibid., 11]. Mais cette exigence de rigueur ne doit pas être circonscrite à l'analyse et à l'arithmétique, car si on n'y soumettait pas également la Géométrie, la Mécanique et la Physique on en viendrait, « comme dernière conséquence », à soustraire de son domaine les concepts du continu et du nombre irrationnel. « Tout au contraire, [...] partout où se présentent des idées mathématiques, soit en Théorie de la connaissance (von erkenntnistheoretischer Seite), soit en Géométrie, soit en Physique, le problème se pose de la discussion des principes fondamentaux, bases de ces idées, et de l'établissement d'un système simple et complet (vollständig) d'axiomes; et cela doit se faire de telle façon que la rigueur des nouvelles définitions et leur applicabilité ne le cèdent en rien aux anciennes définitions arithmétiques. » [ibid., 8]

Pourquoi la méthode axiomatique assure-t-elle une égale rigueur à l'arithmétique, l'analyse, la physique, la géométrie ou la théorie de la connaissance? Et qu'est-ce qui procure à cette méthode l'universalité de son application, une universalité qui fait penser à celle de la $M a$ thesis Universalis du Descartes des Regulae? C'est que, dès lors que se présente une nouvelle idée mathématique, on doit nécessairement lui associer un nouveau signe (Zeichen). Ces signes ne sont pas choisis de manière arbitraire : ils doivent rappeler les phénomènes qui sont à l'origine de la prise en compte des nouvelles idées mathématiques ${ }^{11}$. Or, de ces seules considérations résulte une communauté de nature entre géométrie et arithmétique. «Les signes de l'Arithmétique sont des figures écrites et les figures de la Géométrie sont des formules dessinées ${ }^{12}$. C'est-à-dire : 1) que l'Arithmétique est faite de signes, 2) que ces signes sont là pour d'autres signes : les dessins de la Géométrie, et 3) réciproquement, qu'une formule arithmétique (qui est faite de signes) peut être remplacée par ces autres signes que sont les dessins de la Géométrie ${ }^{13}$. Aucun mathématicien ne pourrait se passer de ces signes que sont les figures géométriques. Mais une méthode rigoureuse de démonstration ne

${ }^{11}$ Ici Hilbert fait penser à Leibniz, mais on ne retrouve pas, dans ses écrits ultérieurs, de considérations explicites à l'appui d'une conformité entre symbolisme et choses symbolisées.

${ }^{12}$ Ibid. En donnant comme traduction de cette phrase : « Les signes et symboles de l'Arithmétique sont des figures écrites, et les formules géométriques sont des formules dessinées », Laugel n'a pas mis les lecteurs de Hilbert sur la bonne voie. Cavaillès a retraduit correctement ce passage [Cavaillès 1938, 93].

${ }^{13}$ L'exemple donné par Hilbert pour ce dernier cas est celui de la double inégalité $a>b>c:$ «... qui ne se sert, dit-il, du dessin de trois points situés l'un à la suite de l'autre sur une droite comme signe géométrique traduisant le mot entre ». 
peut s'appuyer sur ces signes que sont les figures géométriques sans autre forme de procès.

Lorsque nous traitons d'un problème arithmétique dans les conditions usuelles, nous déléguons notre confiance à un certain sentiment arithmétique et à l'efficacité du mode de fonctionnement des symboles arithmétiques. Nous procédons ainsi car nous savons que si nous remontons la chaîne des déductions jusqu'aux axiomes de l'arithmétique cette confiance est justifiée. En effet, c'est en vertu de ces axiomes que nous observons des règles usuelles de calcul comme celle consistant à disposer correctement les chiffres les uns en dessous des autres lorsque nous effectuons une addition. Il en est de même pour les signes de la Géométrie. Si nous ne prenions pas appui sur eux, c'est-à-dire si nous nous privions de notre faculté de voir dans l'espace, nous ne pourrions progresser dans la connaissance géométrique. Et, ici encore, ce qui légitime la manipulation des signes visibles de la Géométrie, manipulation qu'on pourrait dire aveugle si nous la rapportions aux règles de la raison, comme méthode de démonstration, c'est que celle-ci est déterminée par la méthode axiomatique qui permet de dissocier et de combiner les concepts géométriques (c'est-à-dire ces idées mathématiques causées originellement par les phénomènes). La méthode axiomatique est donc la grammaire générale de l'usage des signes associés aux idées mathématiques.

L'empire de la méthode axiomatique n'est pas circonscrit aux différents domaines mathématiques. Cette méthode peut être élevée au rang de discipline des principes et donc, par définition, remplir le rôle d'une philosophie théorique. Pour le voir, revenons sur cette exigence de rigueur, source principale des problèmes qu'on se pose en analyse et en arithmétique, et qui ne répond qu'à un besoin de notre entendement. Si nous l'associons au fait que de très anciens problèmes, comme celui de la quadrature du cercle, « ont reçu une solution parfaitement satisfaisante et rigoureuse, bien qu'en un sens tout différent de celui qu'on cherchait primitivement $»$, elle fait alors naître chez le mathématicien la conviction que tout problème mathématique déterminé « est susceptible d'une solution rigoureuse, que ce soit par une réponse directe à la question posée, ou bien par la démonstration de l'impossibilité de la résolution » [ibid, 11], solution rigoureuse signifiant, derechef, résolution au moyen d'un nombre fini de déductions logiques. De sorte que cette conviction du mathématicien tient le rôle « d'axiome de la résolution de tout problème »[ibid, 11$]^{14}$.

Cette conviction du mathématicien n'est pas limitée aux mathématiques. En effet, avec le $\mathrm{VI}^{\mathrm{e}}$ problème, c'est-à-dire Le traitement mathé-

\footnotetext{
${ }^{14}$ Hilbert postule donc, comme il continuera de la faire au cours de l'élaboration de la théorie de la démonstration, que tout problème mathématique est décidable.
} 
matique des axiomes de la physique, l'axiome de la résolution et les ressources de la méthode axiomatique trouveront leur pleine expression en remplissant cinq rôles distincts. 1) Dissocier les hypothèses, comme en Géométrie. 2) Constituer l'architectonique de la physique en construisant les théories particulières au moyen de l'adjonction de nouveaux axiomes. 3) Permettre au physicien d'avoir égard «non seulement aux théories qui se rapprochent de la réalité, mais encore en général à toutes celles qui sont logiquement possibles » [ibid., 26] et qui, par conséquent, pourraient être amenées à l'existence ${ }^{15}$. 4) Livrer des démonstrations de non-contradiction. Elle préviendra ainsi les physiciens contre « des procédés qui ne sont pas logiquement admissibles dans l'édification rigoureusement logique d'une théorie », tels que l'adjonction, au cours de l'élaboration d'une théorie, d'hypothèses nouvelles justifiées uniquement par l'expérience. 5) Enfin, vérifier si des points de vue opposés au sujet des principes de la Mécanique, comme le sont, par exemple, ceux de Boltzmann et de Mach, le sont réellement ou si, une fois ces conceptions axiomatisées, et au terme d'une démonstration d'équivalence, ces oppositions se révèlent être simplement apparentes [ibid., 25]. Bien que l'application de la méthode axiomatique en physique reste programmatique, la physique nous livre néanmoins des exemples de démonstration d'impossibilité, comme celle de l'inexistence du mouvement perpétuel, avec leurs corrélats positifs, comme ici la découverte du principe de conservation de l'énergie. L'axiome de la résolubilité de tout problème s'applique donc également hors des mathématiques. On peut alors se poser la question suivante : cet axiome est-il propre à la pensée mathématique ou exprime-t-il une loi générale de notre entendement? [ibid., 25] ${ }^{16}$

Puisque les axiomes donnent les définitions des concepts élémentaires de la science et que les systèmes d'axiomes livrent une « description complète et exacte des relations entre les concepts élémentaires de cette science », la question la plus importante que l'on doit se poser à leur égard est de « démontrer qu'en s'appuyant sur les axiomes l'on ne pourra jamais arriver à des résultats contradictoires au moyen d'un nombre fini de déductions logiques. » [ibid., 15]

${ }^{15}$ Plus exactement, elles existent en puissance dès qu'elles sont non contradictoires. Par « être amenées à l'existence» il faut donc comprendre «pourraient dans le futur être employées par les physiciens même si actuellement rien ne laisse supposer la possibilité de cet emploi. »

${ }^{16}$ [Cavaillès 1938] voit, dans ce que Hilbert nomme explicitement un axiome, un principe régulateur. Outre le fait qu'il est plus légitime de suivre la lettre du propos de Hilbert, plutôt que de l'interpréter comme on souhaiterait qu'il soit, c'est bel et bien sa double confiance dans le principe du tiers exclu et dans une forme rudimentaire d'optimisme, comme l'atteste la conception de la théorie physique juste décrite, qu'Hilbert exprime ici. Soit l'exact contraire d'un principe régulateur. 
Si les sciences physiques ne sont pas mûres pour de telles démonstrations, les mathématiques le sont et on peut donner une démonstration de non-contradiction de l'Arithmétique. Cette démonstration doit être directe, c'est-à-dire que, contrairement au cas des démonstrations de cohérence de la Géométrie, on ne peut construire un domaine d'êtres dans lequel une éventuelle contradiction des axiomes de l'Arithmétique serait reflétée. C'est le fameux Second problème de Hilbert. Mais il ne faut pas uniquement voir dans le Second problème la traduction ultime, exprimée sous la forme d'un problème mathématique, de l'exigence de rigueur dans la démonstration. Le second problème vient également à l'appui d'une conception générale de l'être, que Hilbert exposait quelques mois auparavant dans une lettre à Frege, et dont la question formulée à l'instant (l'axiome de la résolubilité de tout problème exprime-t-il une loi de l'entendement?) est le corrélat.

Certains mathématiciens, comme par exemple Kronecker, ont émis des doutes sur l'existence propre des nombres réels. Pour priver ces objections de toute raison d'être, il suffit de fonder le concept d'existence à l'aide de la méthode axiomatique. Lorsqu'on confère à un concept des caractéristiques qui se contredisent, alors ce concept n'a pas d'existence mathématique. Si, par contre, on démontre par l'application d'un nombre fini de déductions logiques que ces attributs ne se contredisent pas, alors l'existence mathématique du concept est démontrée ${ }^{17}$. Exister c'est donc être logiquement possible. Par conséquent «la démonstration de la non-contradiction des axiomes de l'Arithmétique serait en même temps la démonstration de l'existence mathématique de l'ensemble de tous les nombres réels, c'est-à-dire du continu »[ibid., 16]. La méthode axiomatique à qui est délégué l'inventaire des êtres et des non-êtres est ainsi une philosophie théorique, une métaphysique qui se voudrait toute mathématique. Par conséquent, bien que le programme de Hilbert soit de fait constitué d'une liste de problèmes mathématiques - si on excepte l'importante question de la nature de la théorie physique décrite dans le Sixième problème, il constitue également une prise de position sur la nature de la connaissance.

\footnotetext{
${ }^{17}$ Dans la lettre à Frege du 28 décembre 1899 Hilbert écrit : « depuis que je réfléchis à ces sujets, que j'écris et que j'expose sur eux, j'ai toujours dit [. . que] si les axiomes arbitrairement posés avec toutes leurs conséquences ne se contredisent pas, alors ils sont vrais et les choses qu'ils définissent existent. C'est pour moi le critère de la vérité et de l'existence » [Rivenc Rouilhan 1992, 227].
} 


\section{Une doctrine paradoxale de la connaissance}

On peut donc constater que tous les éléments doctrinaux que Hilbert développera postérieurement, au fur et à mesure de l'édification de la théorie de la démonstration, et que les controverses avec Brouwer et Weyl accuseront, sont déjà présents dans la conférence de Paris. Ceci, qu'il s'agisse de la prééminence du signe : «Au commencement - ainsi dit-on ici - est le signe » [Hilbert 1935, 163] [Largeault 1992, 117], du finitisme des déductions [Hilbert 1935, 160] [Largeault 1992, 114], de la possibilité logique comme critère d'existence [ibid.], ou de l'optimisme mécaniciste [Hilbert 1935, 160] [Largeault 1992, 115]. La seule fonction de la méthode axiomatique qui ne soit pas explicitement mentionnée dans la Conférence de Paris est l'ambition de neutraliser les oppositions philosophiques [Hilbert 1935, 163] [Largeault 1992, 117] ${ }^{18}$, c'est-à-dire ce que Hilbert exprimera plus tard ainsi : «Les mathématiques sont une science sans présupposé. Pour les fonder je n'ai besoin ni de Dieu, comme Kronecker, ni de la supposition d'une faculté particulière de notre entendement s'accordant avec le principe d'induction mathématique, comme Poincaré, ni de l'intuition primordiale de Brouwer, ni, non plus, comme le font Russell et Whitehead, des axiomes de l'infini, de réductibilité ou de complétude, lesquels sont en fait des suppositions réelles pourvues de contenu qui ne peuvent être neutralisées par des démonstrations de cohérence » [Hilbert 1927, 85] [Heijenoort 1967, 479].

Cet aspect de la méthode axiomatique ne pouvait se manifester avant que les oppositions philosophiques ne trouvent une occasion pressante de s'exprimer. La découverte des antinomies y pourvoira. Aussi, dans sa première ébauche de la théorie de la démonstration, en 1904, Hilbert se livrera à une revue critique des points de vue des mathématiciens sur les fondements de l'arithmétique. Cet exercice sera un trait constant de tous ses articles ultérieurs consacrés à cette question, à l'exception de Sur l'infini [Hilbert 1925], la plus connue de ses contributions à la théorie de la démonstration.

Soulignons toutefois que cette vertu de neutralité prêtée à la méthode axiomatique l'est déjà en puissance dans la Conférence de Paris. Elle découle immédiatement de la conception de l'existence comme possibilité

\footnotetext{
${ }^{18}$ Cette énumération est limitée aux caractères essentiels de l'hilbertisme. Elle n'est pas exhaustive. On trouve, dès la Conférence de Paris, d'autres thèmes qui seront constamment repris ultérieurement comme celui de l'harmonie préétablie « en un sens différent de celui de Leibniz » [Hilbert 1931] [Ewald 96, 1160], par laquelle Hilbert explique que certains évènements de l'histoire des sciences comme, par exemple, l'antériorité chronologique de la géométrie de Riemann à la Relativité Générale, attestent du fonctionnement parallèle de la Raison et de la Nature.
} 
logique et de la conjecture de solutions positives aux Premier et Second Problèmes.

La thèse de l'universalité de la méthode axiomatique est cependant une thèse philosophique assez singulière, pour ne pas dire paradoxale.

Observons, pour commencer, qu'en proclamant constamment son opposition aux intuitionnistes ou au logicistes, qu'ils soient réalistes ou nominalistes, Hilbert, ne fait que suivre une coutume que l'on trouve chez tous les auteurs défendant des positions philosophiques ${ }^{19}$. Il n'y a là rien de répréhensible tant que ce rituel s'accompagne des autres traits coutumiers de la tribu. Mais certains aspects paradoxaux de la pensée de Hilbert indiquent clairement qu'il ne se plie pas aux usages.

L'aspect paradoxal de la doctrine de Hilbert se manifeste le plus nettement dans ses positions à l'égard de l'infini en acte. Les intuitionnistes et les réalistes reconnaissent ensemble que la notion d'infini en acte ne peut provenir de notre propre fonds. Ils se distinguent en décrétant, pour les premiers, que l'infini en acte n'existe pas, et, pour les seconds, qu'il existe hors de nous. Hilbert se propose de concilier ces deux positions alors que chacune des parties adverses les reconnait clairement comme irréconciliables. Comme les intuitionnistes, il exclut l'infini des méthodes des mathématiques ( «... comme si quelqu'un était jamais parvenu à effectuer un nombre infini d'inférences » [Heijenoort 1967, 370 ] [Largeault 1970, 221]) et, comme les réalistes, il estime cependant que l'on peut connaître cet infini qui, hors de nous et de la Nature, est au Paradis ${ }^{20}$. Ce programme de conciliation revient à ne reconnaître aucune légitimité à l'opposition du réalisme et de l'intuitionnisme. Mais, en même temps, Hilbert reconnaît une part de vérité à l'intuitionnisme : la logique ne permet pas de construire l'arithmétique, et une part de vérité au réalisme : la logique n'est pas stérile. Donnons des axiomes pour les signes de la logique qui n'est pas stérile. Donnons indépendamment, puisqu'ils ne dépendent pas de la logique, des axiomes pour les signes des nombres. Construisons les figures où se mêlent ces deux espèces de signes. Tous les «présupposés », qu'il s'agisse des actions de l'âme des intuitionnistes ou des êtres immatériels des réalistes, ont été éliminés. Les axiomes n'ont saisi que ce qui est essentiel aux mathématiques. L'origine de la connaissance mathématique n'est ni dans l'ego ni dans le concept de totalité infinie.

\footnotetext{
${ }^{19}$ Descartes qui dit du mal d'Aristote ou de Platon, Leibniz qui dit du mal de Descartes, Kant qui dit du mal des rationalistes, etc...

${ }^{20}$ Tout le propos de Sur l'infini invite à prendre en un sens littéral l'image du paradis que Cantor a créé pour nous. C'est la raison pour laquelle [Ladrière 1957] voyait du platonisme chez Hilbert.
} 
Nous l'avons vu, ce qui, pour Hilbert, est un jeu, avec l'axiome de la résolubilité de tout problème, c'est une question concernant la nature de notre entendement. Si on pensait que les propos de Hilbert dépassent sa pensée, que, pour lui, seul compte réellement, pour les deux premiers problèmes, leur solution technique, il faudrait alors expliquer pourquoi Weyl, qui au nom d'un idéalisme constant ${ }^{21}$ avait toujours repoussé les idées de Dedekind et de Cantor, était prêt à rendre les armes quand Hilbert, en 1927, semblait proche de toucher au but. Commentant l'exposé le plus achevé de la théorie de la démonstration que Hilbert ait donné, [Hilbert 1927] [Heijenoort 1967, 464-79], Weyl concluait ses remarques ainsi : «Si les vues de Hilbert l'emportent sur l'intuitionnisme, comme cela semble être le cas, alors j'y verrai une défaite décisive de l'attitude philosophique de la phénoménologie pure, qui montre ainsi son insuffisance pour la compréhension de la création scientifique, ceci même pour le domaine de la cognition le plus primordial et le plus sujet à l'évidence : les mathématiques » [Heijenoort 1967, 484].

Ces trois aspects montrent, à des titres divers, qu'il y a donc un engagement philosophique réel dans l'universalisme de la méthode axiomatique. Mais cet engagement philosophique important, puisqu'il ne s'agit de rien d'autre que de sauver «l'honneur même de l'esprit humain $»^{22}$, se caractérise par deux traits singuliers. Premièrement, c'est un anti-philosophisme puisqu'il prétend dissoudre dans la résolution de problèmes mathématiques ce qu'il y a de spécifique à l'intuitionnisme, au réalisme et même au nominalisme ${ }^{23}$. Cette élimination des présupposés philosophiques est, pour Hilbert, le prix à payer pour garantir la neutralité philosophique de la connaissance mathématique. Elle revient à assigner à l'analyse, et donc à la connaissance de la nature du continu, un statut analogue à celui de l'algèbre. Hilbert ne se contente donc pas de dédaigner les raisons de ses adversaires philosophiques, il fait également fi des leçons de vingt-cinq siècles d'histoire des mathématiques. Deuxièmement, c'est un éclectisme puisqu'il emprunte des éléments à chacune de ces doctrines. La forme particulière de cet éclectisme est assez choquante car elle revient à vouloir engranger, avec les réalistes, les

${ }^{21}$ Le fait que son inspiration ait été variable n'affecte pas la constance de cet idéalisme. Au sujet de la variation de cette inspiration voir dans [Heijenoort 1967] la notice introductive aux commentaires de Weyl sur [Hilbert 1927].

${ }^{22} \mathrm{C}$ 'est Hilbert qui souligne et qui confirme en même temps que «l'élucidation définitive de la nature de l'infini dépasse largement les intérêts d'une discipline particulière »[Hilbert 1925] [Largeault 1970, 222] Cf. supra, note 16.

${ }^{23}$ Comme pour Hilbert le signe est au principe de la connaissance, on peut estimer que sa position présente une grande parenté avec celle de Hanh et Carnap ( $C f$. supra la deuxième remarque de la section 1 ). Mais je ne suis pas parvenu à trouver ce qui pourrait jouer, chez Hilbert, un rôle analogue aux sense data. 
bénéfices de raisonnements où l'infini intervient de façon cruciale en tenant simultanément celui-ci pour un pur rien ${ }^{24}$. L'histoire critique de la théorie de la connaissance montre que les éclectismes ne font jamais long feu. Voyons alors comment ces idées ont pris corps dans le développement du programme.

\section{La recherche du signe de la négation}

La première étape à franchir, dans cette dernière perspective, est de définir les signes pour la logique, puisque c'est par les signes qu'il faut commencer et que, concernant les choses auxquelles la logique s'applique, c'est-à-dire la matière dont est faite l'Arithmétique ou la Géométrie, la cause est, en principe, nous l'avons vu, déjà entendue. Dans la première ébauche de la théorie de la démonstration, [Hilbert 1904], Hilbert va d'abord s'employer à introduire le système de signes recherché pour la logique. Il va ensuite exposer comment ce système pourrait permettre de répondre au second problème en faisant voir que la manipulation des signes représentant les axiomes de Peano ne peut permettre d'engendrer une combinaison de signes qui représenterait une contradiction.

La nature circulaire des raisonnements de Hilbert a été l'objet de critiques précises de la part de Poincarée ${ }^{25}$. Celui-ci reproche justement à Hilbert de s'appuyer sur le principe d'induction pour démontrer le principe d'induction. Poincaré pouvait difficilement demeurer indifférent à l'essai de Hilbert puisque ce dernier s'oppose très directement à un point fondamental de la doctrine de La science et l'hypothèse formulée dès 1894 : le principe d'induction « est le véritable type de jugement synthétique a priori $»$, «ce jugement s'impose (...) à nous avec une irrésistible évidence » car « il n'est que l'affirmation de la puissance de l'esprit »[Poincaré, 1902, 41]. Or cette propriété de l'esprit, Hilbert en fait une propriété de la main qui peut toujours dessiner un signe (un bâ-

\footnotetext{
${ }^{24}$ Les arguments donnés aux premières pages de Sur l'infini pour montrer que l'infini n'existe pas laissent pour le moins perplexe. Faisant allusion au premier modèle cosmologique d'Einstein de 1917 (qui est spatialement fermé), Hilbert nous dit que les « astronomes ont montré que l'univers était fini ». Mais à l'époque ou il écrivait (1925), les controverses, auxquelles participèrent Klein et Weyl, sur l'interprétation géométrique de l'univers de De Sitter battaient leur plein. Par exemple, Lanczos, en suivant une suggestion de Klein, avait donné dès 1922 une interprétation non statique de l'élément linéaire. Sur ces questions, on peut consulter [North 1965, 81-122].

${ }^{25}$ Sur cet aspect, ainsi que sur les prémices de la théorie de la démonstration présentes dans l'essai de Hilbert, voire la notice introductive, ainsi que les notes, de H.Sinaceur à la traduction française de [Hilbert 1904] dans [Rivenc Rouilhan, 245255].
} 
ton) à côté d'une suite de signes (d'une suite de bâtons) donnée. Hilbert partageant, et peut-être empruntant ${ }^{26}$, le point de vue de Poincaré selon lequel on ne peut déduire logiquement le concept de nombre, l'opposition des principes est donc directe et manifeste. Mais la confusion dénoncée par Poincaré compte peu face à celles, difficilement descriptibles, que commet Hilbert dans l'élaboration de la sémiologie de la logique ${ }^{27}$.

En deux mots, et en employant le langage de la logique, ce qui est s'exprimer improprement, Hilbert y confond les expressions bien formées et les théorèmes. Plus précisément, il considère le langage universel $M$ engendré par les deux symboles 1 et $=$, chacun de ces symboles correspondant à 《un objet de pensée ». Il suppose ensuite l'existence d'une partition de $M$ en deux classes complémentaires, celle des êtres et celle des non-êtres. C'est une première difficulté. Hilbert laisse entendre qu'il ne va raisonner que sur la matérialité des signes et qu'il ne sous-entend rien sur leurs référents. Par conséquent, la répartition des mots (formules logiques) en êtres et non-êtres doit relever d'un décret arbitraire qui ne retient rien de l'emploi usuel (mathématique) des nombres entiers et de l'égalité. Mais, pour que cette feinte soit conciliable avec la partition supposée, il faut donc qu'il y ait au moins un critère de forme à l'origine de la partition. Sinon, la distinction entre être et non-être préexisterait à toute grammaire des signes de l'arithmétique, ce qui serait inconciliable avec la doctrine de l'identification de l'être avec la possibilité logique. La nature de ce critère est cependant laissée complètement indécise par Hilbert. Pour comprendre ce que Hilbert ne laisse pas voir, et qu'il devrait indiquer pour que son propos soit simplement interprétable, on peut s'appuyer sur l'analogie avec le langage de la logique. Comme chacun sait, on n'y place pas sur le même plan des expressions telles que $p \vee \neg p$ et $p \neg \neg \neg$. On construit le langage d'une logique pour exclure les assemblages incorrects de signes tels que $p \neg \neg \neg$ et cette construction divise en deux classes disjointes tous les assemblages possibles de signes avec les-

\footnotetext{
${ }^{26}$ Un examinateur de la première version de cet article me reproche de ne pas donner de preuve à l'appui de cette interrogation. Alors faut-il penser que Hilbert n'ait pas lu La sience et l'hypothèse ou qu'il n'en ait pas eu une connaissance indirecte? Le croire reviendrait à prêter à Hilbert un droit assez exceptionnel à l'ignorance, vu 1) le succès du livre de Poincaré, 2) le rang qu'occupaient Hilbert et Poincaré dans la hiérarchie des mathématiciens de leur temps et 3) ce que dit Poincaré dans l'Introduction de La science et l'hypothèse au sujet des axiomes.

Il me semble que la charge de la preuve doit être renversée.

${ }^{27}$ En un sens, elle est également secondaire du point de vue de Poincaré. Pour ce dernier, l'essentiel est, comme il l'a vu tout de suite, que la doctrine de Hilbert fait penser à cette machine légendaire de Chicago bien connue des lecteurs de Tintin en Amérique ou, plus sérieusement, qu'elle requiert la décidabilité de tout problème mathématique.
} 
quels sont construites ces expressions : les expressions bien formées et les expressions mal formées. La division postulée par Hilbert entre êtres et non-êtres, si elle est, est donc du même type que celle entre expressions bien formées et expressions mal formées ${ }^{28}$.

Si $a$ est un mot de $M$, poursuit Hilbert, alors on désigne « également » par $a$ la proposition exprimant que (le mot) $a$ appartient à la classe des êtres et par $\neg a$ la proposition exprimant que $a$ appartient à la classe des non-êtres. Ici on est porté spontanément à s'indigner devant le peu de soin avec lequel Hilbert introduit ses notations. Mais est-il si sûr que, dans la formulation de la logique qu'il a en vue, il ne faille pas identifier le mot $a$ et la proposition $a$ ? Et Hilbert le sait-il lui-même? Quoi qu'il en soit, ou le mot $a$ et la proposition « le mot $a$ appartient à la classe des êtres » sont représentés, dans le langage de la logique, par le même assemblage de signes parce qu'ils doivent être identifiés, et nous avons affaire à une atrocité logique. Ou bien il ne s'agit que d'une maladresse dans le choix des signes (pour les propositions), mais alors celle-ci est de mauvais augure pour une doctrine qui fait de la manipulation réglée des signes le fondement de la théorie de la connaissance.

Au bénéfice du doute, retenons cette dernière possibilité. Remplaçons $a$ par $A$ pour désigner la proposition "le mot $a$ appartient à la classe des êtres" et reprenons le fil du propos de Hilbert. $A$ est vraie, nous dit-il, si $a$ appartient à la classe des êtres et $\neg A$ est vraie si $a$ appartient à la classe des non-êtres. Arrivé à ce point Hilbert estime avoir introduit le signe de la négation logique ${ }^{29}$. Mais appliquons la définition précédente de la vérité à $\neg \neg A$; il vient « $\neg A$ appartient à la classe des (non-êtres qui sont les analogues des) expressions mal formées ». Ce qui est absurde, puisque $\neg A$ n'est pas un mot de $M$ mais une proposition exprimant qu'une certaine combinaison de signes, le mot $a$, n'appartient pas à une classe donnée de combinaisons de signes ${ }^{30}$.

\footnotetext{
${ }^{28}$ Bien entendu ce n'est pas à cette distinction que pense Hilbert puisqu'il range, par exemple, dans les équations homogènes, des expressions mal formées aux côtés d'expressions bien formées. [Hilbert 1904, 262].

${ }^{29}$ [Hilbert 1904, 259] lorsqu'il remarque qu'étant donné que la négation a déjà été introduite, le signe pour ou permettrait de supprimer celui pour s'ensuivre de.

${ }^{30} \mathrm{Un}$ examinateur anonyme de la première version de cet article me reproche de m'appuyer dans cette discussion sur une illusion rétrospective : pour nous, aujourd'hui, la distinction entre un signe et sa référence est claire, mais en allait-il de même à l'époque de Hilbert?

Ignorons les leçons de certains historiens de la grammaire, qui nous apprennent que la théorie saussurienne du signe se trouve déjà chez Aristote, ou celles des historiens des idées, qui nous enseignent qu'avec la constitution de la science et de la philosophie modernes, au XVII ${ }^{\mathrm{e}}$ siècle, s'opère une rupture avec la conception des signes naturels, laquelle institue le signe comme support de la représentation, et tenons-nous-en
} 
Ces remarques suffisent pour comprendre qu'il est utile, lorsqu'on examine la doctrine de Hilbert, de distinguer deux aspects : la thèse générale selon laquelle le signe est au fondement de la logique et des mathématiques et l'exécution proprement dite du projet de la réduction de la connaissance logique et arithmétique à la manipulation de signes. Lorsqu'il revient en 1922, [Hilbert 1935, 157-177] [Largeault 1992, 111-130], dans Les nouveaux fondements des Mathématiques, sur la constitution de la logique, Hilbert écrit ceci :

« Nous n'avons pas introduit d'autres signes logiques que le signe $\rightarrow$ et le quantificateur universel; nous avons notamment évité de formaliser l'opération logique non. Cette façon de traiter la négation est caractéristique de notre théorie de la démonstration; le substitut formel de cette négation absente réside uniquement dans le signe $\neq$, et l'introduction de celui-ci permet d'exprimer et de traiter l'inégalité de façon positive au même titre que l'égalité, bien qu'elle en soit l'opposé. Sur le plan du contenu, la négation n'est employée que dans la démonstration de cohérence, et ceci conformément à nos principes. Eu égard à cette circonstance, notre théorie de la démonstration apporte, me semble-t-il, un éclairage épistémologique important sur la signification et l'essence de la négation. »[Hilbert 1935, 173] Ce qui est exprimé par les axiomes suivants

$$
\begin{gathered}
a \neq a \rightarrow A \\
(a=b \rightarrow A) \rightarrow((a \neq b \rightarrow A) \rightarrow A) .
\end{gathered}
$$

Donc, même si elle prend une toute autre forme, l'ambition présente dans l'article de 1904 demeure. Le changement de forme de l'ambition s'explique par les circonstances nouvelles dans lesquelles s'exprime Hilbert. Il s'agit, pour lui, de mettre un terme aux velléités révolutionnaires de Brouwer et de Weyl, lesquels, si on les écoutait, démembreraient et mutileraient les mathématiques en leur faisant perdre leurs trésors les plus précieux « jusqu'au principe du tiers exclu »[ibid., 160]. Or si on parvient à donner une démonstration de l'applicabilité de ce principe sans s'accorder l'usage explicite de la négation, c'est-à-dire en la remplaçant par des signes d'inégalités entre signes de nombres finis, on coupera à la racine toutes les préventions contre l'emploi du tiers exclu. La démonstration recherchée consiste à simuler dans la théorie de la démonstration

à Hilbert. $1^{\circ}$ ) Celui-ci fait, nous l'avons vu, la distinction, comme les mathématiciens du XVII ${ }^{\mathrm{e}}$ siècle, entre les signes dessinés de la géométrie et ceux, également dessinés, de l'arithmétique. $2^{\circ}$ ) Dans l'essai qui nous occupe, Hilbert vient de faire une distinction entre « objets de pensée » et signes. Faudrait-il excuser Hilbert s'il se prenait à écrire des suites de signes où se mêleraient des dessins de cercles, de nombres, de tangentes, d'opérations arithmétiques...? 
(sans négation) la dérivation des théorèmes critiques de l'analyse, comme celui de l'existence de la borne supérieure. Les chèques signés du tiers exclu seront alors approvisionnés par des démonstrations radicalement constructives.

Cependant quelques mois plus tard [Hilbert 1923], Hilbert propose des axiomes pour la négation, en précisant dans une note que s'il avait évité d'introduire un signe pour non dans son étude précédente, il pouvait maintenant se dispenser de cette précaution car, à la suite d'une modification de sa théorie, l'innocuité de l'emploi de ce signe était assurée. La modification en question, que Hilbert n'indique pas explicitement, réside dans l'introduction de (la première formulation de) l'axiome transfini

$$
A(\tau A) \rightarrow A(a)
$$

et des «axiomes de définition» des quantificateurs qui lui sont associés

$$
\begin{gathered}
A(\tau A) \leftrightarrow(a) A(a) \\
A(\tau \neg A) \leftrightarrow(E a) A(a) .
\end{gathered}
$$

Cette modification de la théorie est accompagnée et complétée par deux axiomes pour la négation

$$
\begin{aligned}
A & \rightarrow(\neg A \rightarrow B) \\
(A \rightarrow B) & \rightarrow((\neg A \rightarrow B) \rightarrow B)
\end{aligned}
$$

qu'il nomme respectivement Principe de non-contradiction et Principe du tiers exclu.

Pourquoi ce changement d'orientation? Le projet initial de donner une définition formelle et constructive de la négation témoignait d'une certaine clairvoyance. Car, après tout, on pouvait voir dans les antinomies des débuts de la logique et dans celles données par Kant le même usage inconditionné de la négation. Mais les analyses de Brouwer laissaient voir qu'une définition constructive de la négation n'aurait pas été compatible avec la loi de la double négation et donc avec le principe $\mathrm{du}$ tiers exclu. C'est ce qui explique la nouvelle orientation choisie par Hilbert : restreindre le champ d'application de la quantification plutôt que celui de la négation.

Dans son article suivant, Sur l'infini, [Hilbert 1925] [Largeault 1970, 220-245], Hilbert introduit directement tous les signes des mots logiques et, ou, si ...alors, non et il donne pour le signe de non les axiomes suivants ${ }^{31}$

$$
(A \rightarrow(B \wedge \neg B) \rightarrow \neg A)
$$

\footnotetext{
${ }^{31}$ Mais également une nouvelle forme à l'axiome transfini : $A(\varepsilon A) \rightarrow A(a)$
} 


\section{$\neg \neg A \rightarrow A \quad$ (Principe de contradiction).}

Cette fois, c'est définitif, la théorie de la démonstration n'ambitionne plus d'apporter un éclairage épistémologique nouveau sur la signification et l'essence de la négation. C'est même tout le contraire : «il faut maintenir en vigueur les règles formellement simples de la logique d'Aristote» [Largeault 1970, 231] que, grâce à cette « harmonie préétablie dont nous constatons si souvent l'action dans l'histoire des sciences », nous trouvons toutes prêtes à l'emploi, sous la forme du calcul logique, pour la théorie de la démonstration [ibid., p.233] ${ }^{32}$.

Il est vrai que, puisque les relations entre les mots tous et il existe manquent totalement de clarté lorsqu'on les applique au domaine des propositions finitistes ${ }^{33}$ on pourrait être tenté « de déterminer les lois

\footnotetext{
${ }^{32}$ Voir supra, note 18.

${ }^{33}$ Ici je dois confier que je suis incapable de comprendre ce que Hilbert entend par tous et il existe appliqués au domaine des propositions finitistes. Avec cette assertion, il prétend récapituler les termes d'une discussion (qui n'est pas encore arrivée à son terme) où il distingue

(i) les propositions concrètes telles que $2+3=3+2$ (où 2 et 3 sont des abréviations pour des assemblages de deux et trois bâtons),

(ii) les propositions telles que $\boldsymbol{a}+1=1+\boldsymbol{a}$ qui sont des jugements hypothétiques et qui, comme tels, ne peuvent être niées et

(iii) les propositions de la forme $a+b=b+a$.
}

Contrairement aux précédentes, les propositions (iii) ne servent pas à communiquer l'identité de deux signes, mais sont des objets formels sans contenu permettant d'obtenir des propositions du type (i) lorsqu'on substitue des numéraux aux lettres $a$ et $b$. De sorte que si les formules de type (iii) n'ont pas de signification, elle permettent néanmoins « de déduire des formules auxquelles nous attribuons une signification en considérant qu'elles communiquent des propositions finitistes ».

Ces distinctions posées, faut-il penser que les propositions de type (ii) appartiennent ou n'appartiennent pas au domaine des propositions finitistes? Après avoir rappelé la différence d'interprétation entre les deux acceptions de "il existe" selon que cette locution s'applique à une disjonction finie ou infinie, et que nous rencontrons le transfini lorsqu'une proposition existentielle ne se laisse pas ramener au premier cas, Hilbert poursuit ainsi : «De la même manière nous avons affaire à une proposition transfinie lorsque nous nions une assertion universelle puisqu'elle s'applique à des numéraux arbitraires. Ainsi, par exemple, la proposition selon laquelle, lorsque $\boldsymbol{a}$ est un numéral, on doit toujours avoir $\boldsymbol{a}+1=1+\boldsymbol{a}$ ne peut, du point de vue finitiste, être niée. C'est ce qui apparaît clairement si nous considérons que celle-ci ne peut être interprétée comme résultant de la combinaison d'un nombre infini d'équations numériques coordonnées par "et", mais plutôt comme un jugement hypothétique assertant quelque chose lorsqu'un numéral est donné.

Il suit en particulier de ceci que nous ne pouvons employer, selon l'esprit du finitisme, la disjonction selon laquelle une équation comme la précédente, dans laquelle apparaît un numéral non spécifié, est soit satisfaite pour tout numéral soit réfutée par un contre-exemple. Car, étant une application du tiers exclu, cette disjonction repose essentiellement sur l'assomption que l'assertion de la validité de cette équation peut être niée » [Heijenoort 1967, 378-9]. 
logiques qui sont valides pour le domaine des propositions finitistes, mais cela ne nous serait d'aucune aide car nous n'entendons nullement renoncer aux lois simples de la logique aristotélicienne» [Heijenoort 1967, 379]. Il faut toutefois justifier l'emploi de ces mots logiques que l'on trouve tout prêts à être utilisés. Si Hilbert a tenté précédemment de faire voir que l'usage de la négation était fondé dans les signes et que, désormais, ce n'est plus le cas, alors d'où peut-elle provenir?

Considérons les propositions finitistes suivantes (les chiffres sont toujours des abréviations de suite de bâtons) :

$$
3>2, \quad 2+3=3+2, \quad 2=3, \quad 1 \neq 1
$$

qui sont directement intelligibles. Elles peuvent être niées, « elles sont vraies ou fausses, on peut opérer librement et sans scrupules sur elles au moyen de la logique classique aristotélicienne; le principe de contradiction vaut pour elles en ce sens qu'une proposition et sa négation ne peuvent pas toutes les deux être vraies; le tiers exclu vaut également pour elles, c'est-à-dire que d'une proposition et de sa négation l'une au moins est vraie. Lorsque je dis : cette proposition est fausse, cela équivaut à : la négation de cette proposition est vraie» [Largeault 1970, 232-233].

Le principe de bivalence ( $A$ est vraie ou $A$ est fausse) est donc le prix d'entrée de l'axiome de la double négation et de l'interdéfinissabilité des quantificateurs que Hilbert associe au principe du tiers exclu ( $A$ ou non $A$ ) comme s'ils ne faisaient qu'un. La justification de l'emploi des signes des mots logiques repose ainsi sur un élément, la vérité, qui jusqu'à présent, et par principe, était exclue de la théorie de la démonstration.

\section{Le calcul des séquents, méthode d'analyse des mots logiques}

Malgré des différences notables de contenu et d'intention, les articles de Hilbert discutés présentent tous un trait commun invariable : Hilbert appelle démonstrations des dessins de la forme

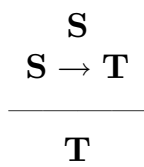

Donc, si on a compris correctement, $\boldsymbol{a}+1=1+\boldsymbol{a}$ n'est pas une proposition finitiste et n'appartient donc pas au domaine des propositions finitistes. Dans ce cas les propositions existentielles et universelles sont exclues de ce domaine et on ne voit plus d'où viennent les difficultés d'usage de la quantification dans le finitisme puisque ne s'y trouvent que des conjonctions et des disjonctions finies. 
et qui, comme tels, tombent immédiatement sous notre sensibilité. Gentzen pousse à son terme cette idée générale de Hilbert en associant à chaque mot logique et à chaque principe démonstratif des dessins de ce type.

Les dessins de Gentzen sont cependant différents de ceux de Hilbert. On peut comprendre pourquoi de la façon suivante. Dans [Hilbert 1922], Hilbert donne pour seuls axiomes logiques ce qu'il nomme les « axiomes de l'inférence logique ». Il reprend ces axiomes quelques mois plus tard dans [Hilbert 1923], mais en les appelant cette fois « axiomes de l'implication (Folge) » et en les complétant, nous l'avons vu, par des axiomes pour la négation. L'intérêt du premier exposé, en se plaçant rétrospectivement du point de vue de Gentzen, est de qualifier ces axiomes avec justesse : ils expriment des propriétés de l'inférence logique; l'intérêt du second exposé est de qualifier avec justesse chacun de ces « axiomes de l'implication » par sa fonction. En cumulant ces intérêts et en trahissant légèrement la lettre du propos de Hilbert en sa faveur, nous avons les : Axiomes de l'inférence logique

$$
\begin{array}{ll}
A \rightarrow(B \rightarrow A) & \text { Adjonction d'une hypothèse } \\
(A \rightarrow(A \rightarrow B)) \rightarrow(A \rightarrow B) & \text { Suppression d'une hypothèse } \\
(A \rightarrow(B \rightarrow C)) \rightarrow(B \rightarrow(A \rightarrow C)) & \text { Echange des hypothèses } \\
(B \rightarrow C) \rightarrow((A \rightarrow B) \rightarrow(A \rightarrow C)) & \text { Elimination d'une proposition. }
\end{array}
$$

Dans les trois premiers axiomes, nous pouvons reconnaître l'analogue des règles structurelles du calcul des séquents, lesquelles portent bel et bien sur les mécanismes inférentiels. A ce sujet, c'est donc la première idée de Hilbert qui était la bonne. Son manque de fermeté dans la désignation correcte de ces axiomes est évidemment une conséquence directe de la confusion entre le concept de conséquence logique et le mot logique $\ll \rightarrow »$, abréviation de $« \neg \ldots \vee \ldots »^{34}$. Or cette distinction est indispensable pour associer des dessins aussi bien aux propriétés de l'inférence logique, énoncées ci-dessus, qu'aux mots logiques. Réintroduisons, avec Gentzen, cette distinction. Nous aurons alors trois concepts auxquels il faudra associer des signes distincts :

- l'inférence dont le signe est -

- la conséquence logique dont le signe est $\vdash$

- le conditionnel dont le signe est $\rightarrow$.

Dans les dessins de Hilbert, il n'y a qu'une relation spatiale entre les signes : celle définie par le signe - Dans ceux de Gentzen il y en a deux : la précédente et celle définie par le signe $\vdash$. Par conséquent les comportements des mots logiques doivent être spécifiés par des dessins décrivant leurs places dans ces relations et, puisqu'il y a deux relations,

\footnotetext{
${ }^{34}$ Frege ne faisait pas cette confusion.
} 
il faut deux dessins par mot logique. Par exemple pour le mot $\rightarrow$, nous avons $^{35}$

$$
\frac{X, A \vdash Y, B}{X \vdash Y, A \rightarrow B} \quad \frac{X \vdash Y, A \quad Z, B \vdash W}{X, A \rightarrow B, Z \vdash Y, W}
$$

Dans la première règle $A$ est à gauche et $B$ est à droite de $\vdash$, dans la seconde c'est l'inverse. Lorsqu'on est dans le premier cas le $\rightarrow$ dessiné en dessous du - l'est à droite, lorsqu'on est dans le second cas, il est dessiné à gauche. On voit alors que décrire la signification et l'essence d'un mot logique revient à décrire sa place dans les deux relations spatiales « de l'autre côté de $\vdash$ »et « de l'autre côté de $-»^{36}$.

Si on se place à nouveau rétrospectivement du point de vue du calcul des séquents, on est très charitable avec Hilbert lorsqu'on rappelle qu'il confond la conséquence logique et le conditionnel. En effet, ce qui est confondu ici ce sont la relation d'inférence, celle de conséquence logique et le mot $\rightarrow$ (puisque Hilbert estime qu'il donne des axiomes pour ce mot). Gentzen donne comme correspondant du premier des axiomes de l'inférence logique les règles suivantes

$$
\frac{X \vdash Y}{X, A \vdash Y} \quad \frac{X \vdash Y}{X \vdash Y, B}
$$

La règle de gauche est bien une règle d'adjonction d'une hypothèse ${ }^{37}$. L'axiome de Hilbert s'obtient avec la démonstration suivante (le dessin suivant)

$\frac{\frac{A \vdash A}{\frac{A, B \vdash A}{A \vdash B \rightarrow A}}}{\frac{A \vdash A \rightarrow(B \rightarrow A)}{\vdash A}}$

\footnotetext{
${ }^{35}$ Dans toutes les règles du calcul des séquents les capitales de la fin de l'alphabet, $X, Y$, etc., désignent des listes de formules séparées par des virgules.

${ }^{36}$ Ceci n'est vrai que pour les mots logiques des calculs propositionnels, nécessaire et possible inclus. (Le cas des modalités est traité par [Scott 1971]). Il faudrait introduire une distinction entre mots logiques analytiques et mots logiques synthétiques pour opposer, d'un côté, ceux qui sont passibles d'une analyse locale en termes de séquents, comme celle que je viens de reproduire pour $\rightarrow$, et ceux qui, comme les quantificateurs, dépendent de liaisons à l'intérieur des démonstrations et privent celles-ci d'une de leur propriété remarquable, à savoir qu'elles forment des objets à deux dimensions.

${ }^{37}$ Celle de droite est une règle d'adjonction d'une conséquence.
} 
où on a appliqué la règle d'adjonction (en remplaçant $X$ et $Y$ par $A$ et $A$ par $B$ ) et deux fois la première règle pour $\rightarrow$. Traduit en mots, ce dessin dit : A est conséquence logique de $A$, d'où on infère que $A$ est conséquence logique de $A$ et $B$, d'où on infère que si $B$ alors $A$ est conséquence logique de $A$, d'où on infère que si $A$ alors si $B$ alors $A$ est conséquence logique d'aucune hypothèse, et donc vaut absolument. De trois inférences et d'une conséquence logique, on a tiré trois conséquences logiques et la démonstration d'une expression logique contenant deux mots logiques. On a analysé complètement l'axiome de Hilbert en dissociant ce qui relève des mots logiques de ce qui relève des propriétés de l'inférence. Chacun des axiomes de Hilbert est passible du même traitement.

Cette méthode, le calcul des séquents, est un progrès par rapport à la méthode axiomatique non seulement parce qu'il permet de dissiper les confusions entre,$- \vdash$, et $\rightarrow$, mais également parce qu'il permet de voir qu'il y a, dans la méthode axiomatique en logique, un parti pris philosophique implicite pour le dogmatisme (au sens de ce terme donné dans la quatrième remarque de la section1).

Hilbert, nous l'avons vu, en est arrivé à invoquer un argument d'autorité pour choisir les mots logiques : ce sont ceux de la logique aristotélicienne, ceux que les hommes emploient depuis qu'ils pensent. C'est là, d'une part, une attitude d'esprit tout à fait étrangère à l'esprit de la méthode axiomatique telle que Hilbert l'a employée en géométrie et, d'autre part, un choix contradictoire avec la fonction épistémologique qu'il prête à cette méthode.

En premier lieu. le mérite de la méthode axiomatique en géométrie consiste précisément dans la dissociation, pour les principes de cette science, de ce qui dépend de ses origines empiriques et de la nature apodictique de sa méthode. L'axiomatique hilbertienne prolonge l'axiomatique euclidienne en même temps qu'elle s'en éloigne. Elle la prolonge en conservant l'idée de l'énonciation préliminaire des principes auxquels les démonstrations doivent se conformer uniformément. Elle s'en éloigne en montrant que l'application de la méthode apagogique est incomplète lorsqu'elle porte sur des notions où l'imagination vient prolonger la sensibilité. En effet, c'est parce que le postulat «par un point extérieur à une droite il ne passe qu'une parallèle » n'est pas univoque, et que cette équivocité dépend de l'imagination qui prolonge indéfiniment la partie visible de la droite, que le libre jeu de l'axiomatique peut s'exercer et mettre à jour l'opposition entre géométries métriques et géométries projectives. 
A l'inverse, poser des axiomes logiques comme $\neg \neg A \rightarrow A$ ou $A \rightarrow$ $A \vee B^{38}$, et donc fixer univoquement les rapports de $\neg$, $\vee$ et $\rightarrow$, c'est éliminer arbitrairement l'indétermination contenue dans la signification usuelle des mots non, ou et si... alors. Cette élimination, qui n'est justifiée que par l'invocation de la nature révélée des mots logiques, est similaire à la confusion des motifs qui conférait à l'axiome des parallèles une valeur catégorique. Le calcul des séquents, contrairement à la méthode axiomatique, ouvre la voie des possibles pour les mots logiques et cette voie contraint les parties en présence (Brouwer et Hilbert), car il pose des couples de règles (dessins) uniques pour chaque mot logique. Les distinctions de sens des mots logiques proviennent des conditions stipulées par les règles structurelles qui concernent les mécanismes inférentiels purs. Il y a un abîme entre les mots logiques des logiques classique et intuitionniste, toute traduction de l'une dans l'autre est une trahison, mais ce n'est là que l'effet direct et profond, invisible dans la méthode axiomatique, des mécanismes inférentiels admissibles. En l'espèce, c'est le rejet de la règle d'adjonction d'une conséquence, donnée plus haut, qui rend compte de l'inacceptabilité des principes du tiers exclu et de la double négation pour les intuitionnistes. Les mots de la logique intuitionniste ne sont pas plus mystérieux que d'autres, ce sont ceux donnés par les règles logiques communes à tous les systèmes logiques. Le pluralisme géométrique est justifié par la méthode axiomatique. La réalité historique du pluralisme logique est fondée par une théorie des signes des mots logiques qui requiert l'abandon de la particularisation de l'interprétation des mots logiques qu'implique inévitablement l'emploi de la méthode axiomatique.

En ce sens, le calcul des séquents, avec ses deux relations d'échange de position données plus haut, est à la logique ce que la théorie des groupes de déplacements rigides est à la géométrie métrique synthétique.

En second lieu, puisque Hilbert a renoncé à voir dans les mots logiques des signes sui generis, il faut indiquer à quoi correspondent ces mots et, donc poser une notion de vérité extérieure aux signes à laquelle leur emploi doit se conformer. Autant Hilbert aurait pu éviter, dans sa brève justification de l'emploi de la « logique aristotélicienne », de mêler le tiers exclu et le principe de bivalence, autant il ne pouvait éviter, pour légitimer la méthode axiomatique, une fois reçu des Cieux ou de l'autorité d'Aristote le cadeau des mots logiques, de faire dépendre du vrai et

\footnotetext{
${ }^{38}$ A partir de Sur l'infini, Hilbert donne également des axiomes pour la disjonction et la conjonction. Ceux-ci ne sont pas mentionnés explicitement dans cet article car Hilbert ne donne que des échantillons des axiomes logiques. On trouve la liste exhaustive de ces axiomes dans son article suivant [Hilbert 1927].
} 
du faux la signification et l'essence de la négation. Cette distinction entre énoncé et état de chose, propre au dogmatisme, est complètement étrangère à l'esprit du constructivisme, qu'il s'agisse de celui de Hilbert ou de celui de Brouwer. Elle s'oppose explicitement à la doctrine de l'existence comme possibilité logique telle qu'elle est exprimée dans la lettre à Frege et, en dernier ressort, elle est contradictoire avec une éventuelle réponse positive au Second Problème et, donc, avec le but même de la théorie de la démonstration.

\section{Conclusion : Applications des remarques précédentes}

Pour que l'histoire puisse nous éclairer sur le présent, ce qui est la seule chose qu'on puisse en attendre, il faut cesser de la confondre avec l'hagiographie. A peine formulées, les idées de Hilbert ont été jugées ineptes par certains de ses contemporains qui étaient des penseurs exigeants. Hilbert ne s'en est pas soucié. Un peu plus tard, deux théorèmes de Gödel ${ }^{39}$ ont prouvé mathématiquement l'absurdité de ces idées. Il n'empêche. Le scientisme, maladie sénile de la science, a repris le dessus $^{40}$.

L'analyse historique de cet événement majeur de l'histoire de la logique qu'a été le programme de Hilbert mérite pourtant mieux que cela car, pour la seule question, très limitée, de la négation logique, elle est riche d'enseignements pour la pensée contemporaine. On peut même dire que si cet épisode de l'histoire de la logique était intégré à l'enseignement de la logique mathématique, la pensée contemporaine ne serait pas tombée dans deux travers qui ont dominé la recherche en philosophie et en informatique au cours des dernières décennies.

Il y a une première parenté entre la doctrine de Hilbert et celle de Quine : cette dernière est également un éclectisme [Vuillemin 1986]. Quine, contrairement à beaucoup de ses pseudo disciples, ou pseudo adversaires, sait que les mathématiques actuelles sont réalistes, que le théorème d'incomplétude mine irrémédiablement toute doctrine empiriste de la connaissance et qu'accepter le théorème de complétude de

\footnotetext{
${ }^{39}$ Le théorème de complétude de la logique classique du premier ordre et le théorème d'existence de propositions arithmétiques formellement indécidables.

${ }^{40}$ Comme on l'a vu plus haut (quatrième remarque de la section 1) avec la citation de Bourbaki. J'ai essayé de montrer ailleurs, [Audureau 2004, 110-114], qu'à suivre Bourbaki à la lettre, la logique mathématique aurait dû se priver du théorème de complétude. Ce qui ne manque pas de sel de la part de ce contempteur de l'intuitionnisme.
} 
la logique classique revient à adhérer au réalisme. Pour sauver ce qui pouvait l'être de l'empirisme, il a donc élaboré une doctrine de la logique classique qui présente une seconde parenté avec celle de Hilbert. Alors que Hilbert voulait faire sortir la négation de la logique classique des signes écrits, Quine a voulu la faire sortir des signes sonores. Mais, comme on le sait, Quine s'est heurté à des difficultés insurmontables qui sont analogues et parallèles à celles rencontrées par Hilbert. 1) Il voulait montrer que la logique classique, et seulement elle, est immanente au comportement verbal, mais il a dû concéder que la logique intuitionniste pouvait tout autant revendiquer cette prétention, 2) Sa tentative de faire sortir la négation logique du dissentiment n'échappe pas, et ne pouvait échapper, au paralogisme. 3) Sa définition de la satisfaisabilité par les suites, qu'il prétend réductible à l'arithmétique finitiste, repose sur l'identification d'un individu avec le singleton contenant cet individu et, donc, sur l'identification du particulier et de l'universel. Ces trois difficultés ne sont toutefois que les conséquences, parmi d'autres, d'un tour de passe-passe si bien réussi qu'il est demeuré complètement inaperçu. Affirmant rapidement, comme s'il ne valait même pas la peine d'y penser, qu'il y a une grammaire, au sens d'un système de réécriture, pour le langage de la logique classique du premier ordre, il s'est dispensé d'en donner les règles ${ }^{41}$. En souhaitant bon courage à qui entreprendrait d'en rédiger les règles, contentons-nous d'observer que cette prétention rapproche la doctrine de la logique de Quine de celle de Hilbert encore plus que l'analogie entre la forme écrite et la forme verbale du signe.

L'un des problèmes qui a dominé la recherche en intelligence artificielle au cours des trois dernières décennies consistait à rechercher une interprétation procédurale de la négation de la logique classique. Cette question s'est présentée lorsqu'on a voulu employer le langage de cette logique comme un langage de programmation. Les travaux qui lui ont été consacrés furent si nombreux qu'il a fallu créer plusieurs magazines spécialisés pour les accueillir. Comme on pouvait s'y attendre, le concept de vérité de la logique classique étant « hautement transfini » ${ }^{42}$ et la négation de la logique classique étant liée indissociablement à ce concept, ces travaux se sont soldés par un échec, car les ordinateurs, eux, ne peuvent effectivement manipuler que la forme matérielle des signes des mots logiques.

\footnotetext{
${ }^{41}[$ Quine 1973, 19] affirme plus que cela : la grammaire de la logique est une grammaire de constituants, elle n'a pas besoin de règles transformationnelles. Sur ces questions [Audureau 2000].

${ }^{42}$ L'expression est de Gödel in [Wang 1974, 8].
} 
Ces deux exemples permettent de voir pourquoi l'étude historique des erreurs de Hilbert peut être utile au développement présent de la connaissance positive.

\section{Bibliographie}

Audureau, Eric

2000 Les objets de la logique classique peuvent-ils être des énoncés?, Philosophiques, 27, 263-85.

2004 Kurt Gödel, critique de la théorie de la relativité générale, Thèse de doctorat de l'Université de Provence.

\section{Bourbaki, NicOlas}

1960 Éléments d'histoire des mathématiques, Paris : Hermann.

CAVAillès, JeAn

1938 Méthode axiomatique et formalisation, Paris : Hermann.

Curry, Haskell

1977 Foundations of Mathematical Logic, Dover Books.

EWALD, WiLliam

1996 From Kant to Hilbert, A Source Book in the Foundations of Mathematics, Vol. II, Clarendon Press.

Gentzen, Gerhard

1963 The collected works of Gerhard Gentzen, ed. by Szabo, M., North-Holland.

GöDEL, KuRT

1929 Über die Vollständigkeit des Logikkalküls in Kurt Gödel Collected Works, vol.1, ed. S. Feferman, 60-100, New York : Oxford University Press.

1930 Die Vollständigkeit der Axiome des logischen Funktionenkalküls, Monatshefte für Mathematik und Physik, 37, 349-360.

1931 Über formal unentscheidbare Sätze der Principia Matematica und verwandter Systeme I, Monatshefte für Mathematik und Physik, 38, 173-198.

HiLBERT, DAVID

1902 Sur les problèmes futurs des mathématiques, Paris : GauthierVillars. 
1904 Über die Grundlagen der Logik und der Aritmetik, Verhand. des 3. internat. Math. Kongr. in Heidelberg, Teubner, 1905. Cité d'après [Rivenc Rouilhan 1992].

1922 Neubegründung der Mathematik, Abhandlungen aus dem mathematischen Seminar der Hamburgischen Universität, 1, 157-177. Cité d'après [Hilbert 1935, 157-177] et d'après la traduction française par [Largeault 1992, 111-130].

1923 Die logischen Grundlagen der Mathematik, Mathematische Annalen, 88, 151-165. Cité d'après [Hilbert 1935, 178-191] et d'après la traduction française par [Largeault 1992, 131-144].

1925 Über das Unendldliche, Mathematische Annalen, 95, 1926. Cité d'après la traduction anglaise de [Heijenoort 1967] et la traduction française par [Largeault 1970, 220-245].

1927 Die Grundlagen der Mathematik, Abhandlungen aus dem mathematischen Seminar der Hamburgischen Universität, 6, 65-83. Cité d'après la traduction anglaise par [Heijenoort 1967] et la traduction française de [Largeault 1992, 146-163].

1928 Probleme der Grundlegung der Mathematik, Mathematische Annalen, 104, 1-9, 1929. Cité d'après la traduction française par [Largeault 1992, 177-185].

1930 Naturerkennen und Logik, Die Naturwissenschaften, 18, 959963. Cité d'après la traduction anglaise par [Ewald 1996, 11571165].

1931 Die Grundlengung der elementaren Zahlentheorie, Mathematische Annalen, 104, 485-94, 1931. Traduction française par [Largeault 1992, 188-196].

1935 Gesammelte Abhandlungen, Bd. III, Springer, 1935. Réimp., Chelsea.

VAn HeiJenoort, Jean

1967 From Frege to Gödel, A Source Book in Mathematical Logic, 1879-1931, Harvard University Press.

LADRIÈRE, JEAN

1957 Les limitations internes des formalismes, Paris : Gauthier-Villlars, 1957 ; réimpression, Paris : Jacques Gabay.

LARGEAULT, JEAN

1970 Logique mathématique. Textes, Paris : A. Colin.

1992 Théorie de la démonstration, Paris : Vrin. 
Mancosu, PaOlo

1999 Between Vienna and Berlin : the immediate reception of Gödel's incompleteness theorems, History and philosophy of logic, 20, $33-45$.

NoRTH, JOHN DAVID

1965 The Measure of the Universe, Oxford University Press, 1965; rééd. Dover Books, 1990.

Padoa, Alessandro

1902 Le problème $\mathrm{n}^{\circ} 2$ de M. David Hilbert, L'enseignement mathématique, 1903, 5, 505-527.

Poincaré, Henri

1902 La science et l'hypothèse, Paris : Flammarion, 1968.

1905 La valeur de la science, Paris :Flammarion, 1970.

Quine, William van ORMaN

1973 Philosophie de la logique, Paris : Aubier.

Rivenc, François et de Rouilhan, Philippe

1992 Logique et fondements des mathématiques, Paris: Payot.

SinACEUR, Hourya

1992 Notice introductive à [Hilbert 1904] dans [Rivenc et Rouilhan 1992], 245-255.

Vuillemin, Jules

1986 What are Philosophical Systems?, Cambridge University Press. HAO WANG

1974 From Mathematics to Philosophy, Routledge \& Kegan Paul. 